\section{Transient and sudden hypotension with use of fibrin glue!}

\section{Ghansham Biyani, Charu Mahajan, Indu Kapoor, Neeraj Kumar}

We report a case of a 9-year-old boy diagnosed with aneurysmal bone cyst (ABC) of C5 to D1 vertebral levels and scheduled for re-exploration and tumour excision under general anaesthesia. He was apparently healthy 5 years back when he developed progressive weakness of both lower limbs and a sense of incomplete evacuation of bladder. He was operated for the tumour excision along with rod placement and pedicle screw fixation without any complications. He was re-explored 2 years later for the recurrence of ABC. For the past 2 months, he had been complaining of pain at the surgical site associated with decrease in the motor power in the upper and lower limbs. Magnetic resonance imaging (MRI) revealed residual ABC causing cervico-dorsal canal stenosis with cord changes between C6 to D2 vertebral levels. Routine blood investigations were within normal limits.

General anaesthesia was induced with fentanyl $80 \mathrm{mcg}$, propofo $170 \mathrm{mg}$ and rocuronium $40 \mathrm{mg}$. Besides routine monitoring, invasive monitoring (arterial and central venous cannulation) was performed. Owing to multiple surgeries in the past, adhesions and fibrosis were present at the surgical site, resulting in excessive oozing of blood. Surgeons applied fibrin sealant (TISSEEL ${ }^{\mathrm{TM}}$ KIT) to achieve haemostasis. Immediately the patient had sudden hypotension (blood pressure decreased from $110 / 70$ to $50 / 22 \mathrm{mmHg}$ ), without any change in the heart rate [Figure 1]. The plethysmography curve also disappeared, possibly because of low peripheral perfusion. The surgeons were informed immediately. Fluid resuscitation was done with crystalloid $(500 \mathrm{ml})$ and colloid $(500 \mathrm{ml})$. Mephenteramine $6 \mathrm{mg}$ was administered along with hydrocortisone $100 \mathrm{mg}$. Blood pressure increased to $110 / 64 \mathrm{mmHg}$ in the next few minutes and surgery was resumed. The rest of the intraoperative and postoperative courses were unremarkable.

In our case, there remains a possibility of hypersensitivity reaction to fibrin sealant in paediatric patient undergoing spine surgery in prone position. It is likely that the hypersensitivity to fibrin glue resulted in sudden haemodynamic instability. Fibrin sealants (fibrin glue/fibrin adhesive) are the plasma-derived products, which mimic the final stage of clotting pathway resulting in the conversion of fibrinogen to fibrin. These products have haemostatic properties and act independent of patients' clotting mechanism in the body. These products are either homologous or autologous in origin. ${ }^{[1]}$ They are used to achieve haemostasis to seal tissue and to support wound healing. ${ }^{[2]}$ However, the use of these products is not free from complications. Severe and fatal incidents of anaphylactic reactions and thromboembolism have been reported in literature ${ }^{[3-5]}$ The incidence of hypersensitivity reactions is extremely rare, ranging from 0.5 per 100,000 cases for all reactions to 0.3 per 100,000 cases for serious reactions. ${ }^{[6]}$ The serologic screening includes qualitative and quantitative estimation of aprotinin specific IgE and IgG antibodies. ${ }^{[7-9]}$ Fibrin sealants contain human protein concentrate (fibrinogen, plasminogen and factor XIII), thrombin, aprotinin and calcium chloride. It is contraindicated in individuals with a known hypersensitivity to aprotinin. In few cases, these reactions have also progressed to severe anaphylaxis. [4-6] Such reactions are seen when fibrin sealants are applied repeatedly over time or in the same sitting. Koberand colleagues reported anaphylactic reaction due to systemic administration of aprotinin where it was used for coronary artery bypass graft surgery. ${ }^{[10]}$ In another case, mortality was reported because of the application offibrin glue on adural tear. ${ }^{[11]}$ Here, it is important to state that even if the first contactis well tolerated, it does not exclude the occurrence of an immediate or a delayed allergic reaction. ${ }^{[7,8]}$ The signs and symptoms include bradycardia, tachycardia, hypotension, flushing, bronchospasm, wheezing, dyspnoea, nausea, urticaria, angioedema, pruritus, erythema and paraesthesia. Mild reactions can be managed with antihistaminic, steroids and adrenaline. The serologic screening includes qualitative and quantitative estimation of aprotinin-specific IgE and IgG antibodies, skin prick tests for bovine aprotinin and drug-induced lymphocyte stimulation test..$^{[-9]}$ However, in the postoperative period, we did not investigate our patient for antibodies specific for aprotinin, as the patient refused to give consent for further investigations. Incidence of embolism hasalso been reported with fibrin glue, hence it should not be injected directly into the circulatory system. ${ }^{[4]}$ To avoid adverse life-threatening anaphylactic reactions,

Department of Neuroanaesthesiology, All India Institute of Medical Sciences, New Delhi, India

Address for correspondence:

Dr. Indu Kapoor, Department of Neuroanaesthesiology, Neurosciences Centre, All India Institute of Medical Sciences, New Delhi - 110029 , India.

E-mail:dr.indu.me@gmail.com 


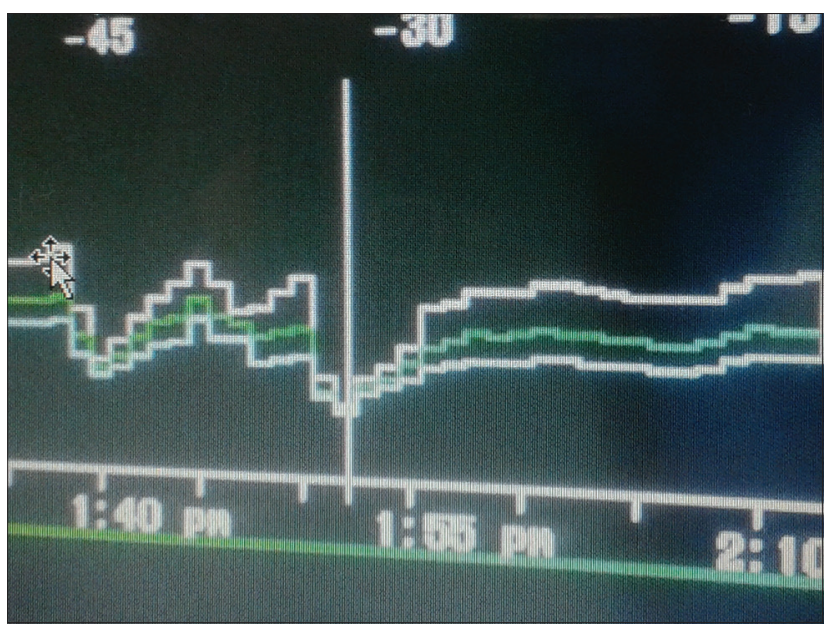

Figure 1: Graph showing sudden fall in Arterial blood pressure. Upper white line- systolic blood pressure green line- mean arterial blood pressure lower white line- diastolic blood pressure

any treatment with aprotinin during the previous 12 months should be considered as a contraindication, as recommended in the Food and Drug Administration guidelines. ${ }^{[12]}$

Our case is a reminder of a possible hypersensitivity reaction with use of fibrin sealants. As we have discussed above, the hypersensitivity reaction could range from mild reaction to mortality. Hence, it should be used very cautiously and judiciously in neurosurgical practise.

\section{REFERENCES}

1. Dhillon S. Fibrin sealant (evicel ${ }^{\circledR}$ [quixil ${ }^{\circledR} /$ crosseal $\left.^{\mathrm{TM}}\right]$ ): A review of its use as supportive treatment for haemostasis in surgery. Drugs 2011;71:1893-915.
2. Fattahi T, Mohan M, Caldwell GT. Clinical applications of fibrin sealants. J Oral Maxillofac Surg 2004;62:218-24.

3. Mitsuhata H, Horiguchi Y, Saitoh J, Saitoh K, Fukuda H, Hirabayasi Y, et al. An anaphylactic reaction to topical fibrin glue. Anesthesiology 1994;81:1074-7.

4. Berguer R, Staerkel RL, Moore EE, Moore FA, Galloway WB, Mockus MB. Warning: Fatal reaction to the use of fibrin glue in deep hepatic wounds. Case reports.J Trauma 1991;31:408-11.

5. Orsel I, Guillaume A, Feiss P. Anaphylactic shock caused by fibrin glue. Ann Fr Anesth Reanim 1997;16:292-3.

6. Kon NF, Masumo H, Nakajima S, Tozawa R, Kimura M, Maeda S. Anaphylactic reaction to aprotinin following topical use of biological tissue sealant. Masui 1994;43:1606-10.

7. Hayashi K, Nagano J, Hattori S. Adhesive arachnoiditis after percutaneous fibrin glue treatment of a sacral meningeal cyst. J Neurosurg Spine 2014;20:763-6.

8. Kanazawa R, Sato S, Iwamoto N, Teramoto A.Allergicreaction following arachnoid plasty with a fibrin sealant. Neurol Med Chir (Tokyo) 2010;50:608-10.

9. Shirai T, Shimota H, Chida K, Sano S, Takeuchi Y, Yasueda H. Anaphylaxis to aprotinin in fibrin sealant. Intern Med 2005;44:1088-9.

10. Kober BJ, Scheule AM, Voth V, Deschner N, Schmid E, Ziemer G. Anaphylactic reaction after systemic application of aprotinin triggered by aprotinin-containing fibrin sealant. Anaesth Analg 2008;107:406-9.

11. Oswald AM,Joly LM, Gury C,Disdet M,Leduc V,Kanny G.Fatal intraoperative anaphylaxis related to aprotinin after local application of fibrin glue. Anaesthesiology 2003;99:762-3.

12. Levy JH, Adkinson NF Jr. Anaphylaxis during cardiac surgery: Implications for clinicians. Anesth Analg 2008;106:392-403.

\begin{tabular}{|l|l|}
\hline \multicolumn{2}{|c|}{ Access this article online } \\
\hline Quick Response Code: & Website: \\
\hline & www.jnaccjournal.org \\
\cline { 2 - 2 } & \\
\hline
\end{tabular}

